“C 2017 IEEE. Personal use of this material is permitted. Permission from IEEE must be obtained for all other uses, in any current or future media, including reprinting/republishing this material for advertising or promotional purposes, creating new collective works, for resale or redistribution to servers or lists, or reuse of any copyrighted component of this work in other works." 


\title{
A Novel Design Procedure for Designing Linear Generators
}

\author{
O. Farrok, M. R. Islam, Y. G. Guo, J. G. Zhu, and W. Xu
}

\begin{abstract}
A number of research works have been carried out based on the optimization of linear generators for harvesting oceanic wave energy, but no significant method of graphical optimization for determination of the shape of linear generator has been found. Moreover, inclusion of some parameters, such as shape of linear generator's translator and stator, has been considered out of the scope of the conventional, adaptive or knowledge based genetic algorithm. This paper proposes a novel method through which any type of linear generator's shape can be optimized graphically. A mathematical model of the proposed method including human intervened genetic algorithm is presented. The proposed method has been applied to a direct drive system based linear generator where the maximization of electrical power output and minimization of steel core volume have been considered as the optimization objectives. The optimization parameters have been further optimized graphically within functional volumetric and electromagnetic constraints to achieve improved design solutions. The proposed method has included comprehensive geometric dimensions, magnetic and electrical parameters. Finally, the shape of steel cores of the translator and special $\mathrm{m}$-shaped stator of the linear generator is determined and simulated both for copper wire and superconductors. This optimized shape of the linear generator is capable of satisfying the multiobjectives of maximal electrical power generation and reduction of its size. The ANSYSIAnsoft software has been used to create the platform for analyses of the whole system.
\end{abstract}

Index Terms-Linear generator, $\mathrm{m}$-shaped stator, shape optimization, wave energy converter.

\section{INTRODUCTION}

$\mathrm{N}$ OWADAYS the electrical energy generation cost, obtainability and ecological safety are remarkable issues that have driven scientists to make the obvious use of renewable energy resources (RERs) like wind [1], [2], solar [3], and hydro power [4]. As a result, numerous researches have been commenced to improve the technology for the conversion of different RERs [5]-[7], including the oceanic wave energy (OWE) [8]-[10]. Oceanic wave has high power density and feasible commercial aspect. A wide variety of oceanic wave energy converters (OWECs) such as Archimedes wave swing, Pelames structure, and oscillating water column, have been planned, manufactured and analyzed by different individuals and institutions. The function of oceanic wave energy devices (OWEDs) is to capture the mechanical power from the sea and convert it into electrical power.

The existing power take off (PTO) systems with high speed rotation electrical machines used as generator, can be connected directly to the electrical grid and have been used in many of the OWEDs. The linear generator has been considered one of the promising techniques for mechanical PTO system either directly joined to a floating buoy, or via some mechanical connection in the OWEC. This arrangement is known as direct drive system (DDS) which is strategic for eliminating intermediate devices to reduce the system complexity and improve the system reliability. As the DDS is directly tied to the linear generator, its induced voltage in the winding depends on the slow and periodic motion of the driving oceanic wave. Therefore, the linear generator generates electrical power with variable magnitude and frequency due to the intermittent nature of the oceanic waves. This variable ac voltage is converted into dc voltage and then converted into ac voltage with constant magnitude and frequency by means of a power electronic converter. To increase the energy conversion efficiency, the power drivers working together with the OWEC are needed to deal with the vertical component of the wave motion.

A graphical optimization technique for an iron-less and brush-less permanent magnet generator has been presented in [11]. The design optimization guidance has been implemented in [12] with some vital parameters and the influence of the Halbach arrays of permanent magnets have been observed. Different projected gradient optimization schemes for a point absorber type PTO system have been formulated in [13]. The optimization of an immersed PAT OWED and the Torres OWED have been discussed in [14] considering the maximum generation of electrical power.

A novel linear air-core topology has been presented in [15], which eliminates most of the end effects associated with the longitudinal flux iron-cored machines as well as the attraction forces between iron-cored stators and magnet translators. The optimization of a tubular permanent magnet linear generator (PMLG) related to the sea wave energy generation for small sensorized buoy was presented in [16], which was developed by means of a hybrid evolutionary algorithm. An external tubular linear permanent magnet generator was proposed in [18] for the DDS for wave energy conversion. Among various design topologies, a flux-switching PMLG can be treated as very effective and low cost OWEC [17]. To investigate the electromagnetic characteristics and the unique advantages of the generator proposed in [18], an optimized tubular secondary PMLG is compared with the tubular primary PMLG. A novel 
flux-switching PMLG was proposed for wave-energy extraction in [19] and it is found that the optimal structure of the linear generator has advantages of better voltage waveform and lower cogging effect. The designs and optimization methods of flux switching PMLG presented in [19] is highly noticeable because they meet nearly all the features of a promising linear generator as OWEC. Nevertheless, some significant changes in shapes of these linear generators' designs can vividly increase the electrical power generation capability and overall performance.

An optimization algorithm has been used analytically in [20] to maximize the apparent airgap power transferred under tangential stress constraint. Another analytical algorithm has been presented in [21] for an external rotor machine design to calculate the main dimensions of the stator and rotor lamination. The speed improvement for optimizing threephase electrical machine designs has been presented in [22]. The nonlinear modeling of the torque of the brushless permanent magnet synchronous motor by using symbolic regression was presented in [23] where an optimized algebraic equation for modeling the machine behavior was derived using genetic programming. The approach of the cheapest designs that fulfill the IE-2 and IE-3 requirements, respectively, has been pointed out in [24] by cost optimization for two different types of synchronous machines with $3 \mathrm{~kW}$ of rated power and $1500 \mathrm{rpm}$ of nominal synchronous speed. The analysis, optimization and design of a two-pole dc brush-less limited-angle torque-motor with a toroidally wound armature using magnetic equivalent circuit modeling was presented in [25].

An initial approach for the graphical optimization of linear generators has been explained in [26]. It is analyzed that the only variations in iron core shapes of the linear generator dramatically change the electrical power generation considering all other parameters being the same. A thorough literature survey was conducted and there is no paper that has focused on the physical shapes.

This paper presents the concept of the shape optimization of a linear generator for the first time so far. A chronological design procedure for the graphical shape optimization is presented using a human intervened genetic algorithm (HIGA). The design effectiveness and performance of a flux switching PMLG using the proposed design procedure are simulated to fulfill the objective. Performances are compared with the existing one presented in [19], which has been claimed novel and optimized. The curvature of the proposed m-shaped stator of the flux switching PMLG has been defined by composite Bezier curve for the shape optimization.

\section{Optimization Problem of the Linear Generator}

In the existing genetic algorithm, some of the parameters for optimization, such as pole-tooth gap, length, pitch, depth, and air gap length, have been considered. These parameters have been determined within a specific range for optimization. The cross sectional view (front) of an existing promising flux switching PMLG made of steel cores and copper coils is shown in Fig. 1. The cross sectional view exposes the core shape of the flux switching PMLG sketched on a plane. The translator core actually travels vertically in the sea although it is seen horizontal according to Fig. 1. The North and South poles of the permanent magnet are denoted by $\mathrm{N}$ and $\mathrm{S}$, respectively. The conventional genetic algorithms used for electrical machine optimization are basically followed by some steps. These steps are initialization of populations, evaluation by fitness function, reproduction process, new generation, end check, final result or evaluation step, and repeat the steps depending on the outcomes.

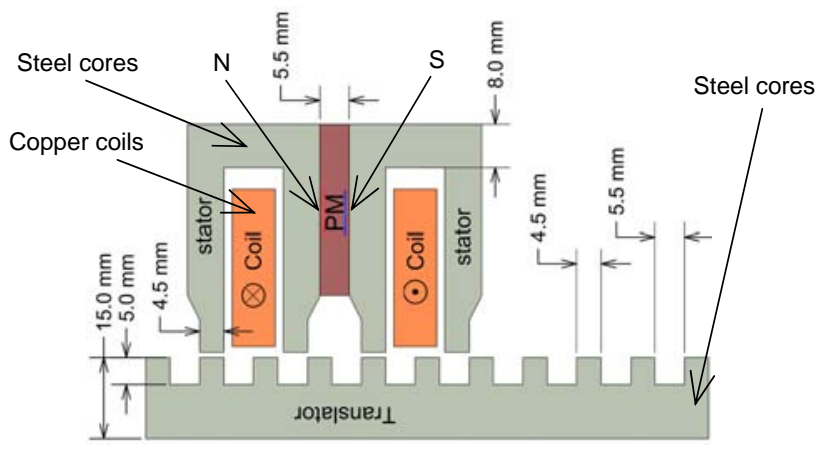

Fig. 1. Construction of the existing flux switching PMLG.

The existing genetic algorithms deal with a single or multiobjective fitness/optimization function. The application of the existing genetic algorithm suffers from the fact that, it is long time consuming if it is used for shape optimization. Now the enquiry arises about the probable number of core shapes and the necessary time duration to find the optimum solution. In fact, there are unlimited numbers of possible shapes and endless time is required for the optimization function to converge if the existing genetic algorithm is applied. Therefore, it is desirable to find out an effective method to achieve the possibly quickest solution in selecting an appropriate shape and size of the stator and translator cores. Such a method has been reveled in this paper because the conventional optimization methods are beyond the scopes of shape optimization in reality.

The goal is to determine a better design within the probably lowest time interval. The proposed method optimizes the parameters of the linear generator quickly with less number of populations and generations. It minimizes the convergence time for optimal solution and is suitable for determining optimal geometry of the linear generator to maximize the output power with minimum size.

\section{Design Analysis of the Existing PMLG}

The architecture of an optimized flux switching PMLG presented in [19] is considered as a reference in this paper. Fig. 2 shows the translator core's isometric view where the slots, teeth, depth, stroke length, and translator length have been indicated. The magnetizing curve of the mild carbon steel used in designing the core of the flux switching PMLG is shown in Fig. 3(a). Fig. 3 (b) shows the specific core loss with an excitation frequency of $50 \mathrm{~Hz}$. The voltage frequency is 50 $\mathrm{Hz}$ for $1 \mathrm{~m} / \mathrm{s}$ speed of the translator. To make a fair comparison, the same parameters have been considered for 
both the existing and the proposed flux switching PMLGs as specified in Table I.

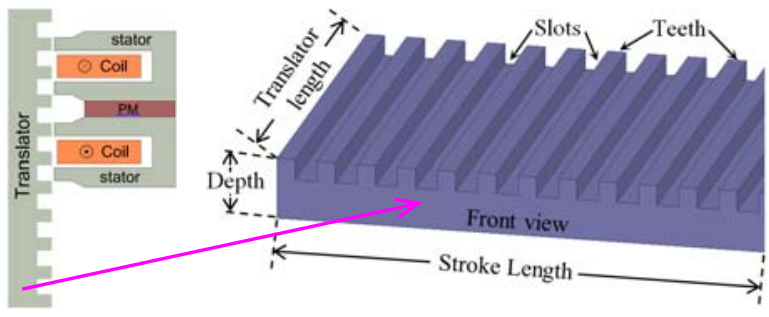

Fig. 2. Isometric view of the tanslator.

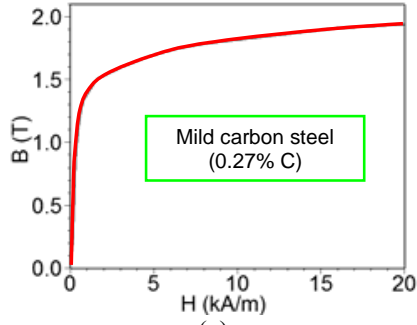

(a)

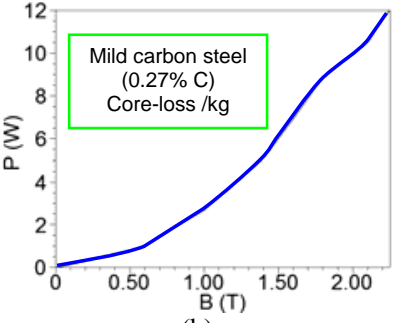

(b)
Fig. 3. (a) Magnetizing curve, and (b) core loss profile of the iron core.

TABLE I

COMMON PARAMETERS FOR THE EXISTING AND PROPOSED FLUX SWITCHING PMLGS

\begin{tabular}{lc}
\hline \hline \multicolumn{1}{c}{ Name of the parameter } & Value \\
\hline Stator length $(\mathrm{mm})$ & 100 \\
Thickness of permanent magnet $(\mathrm{mm})$ & 5.5 \\
Stator tooth width $(\mathrm{mm})$ & 4.5 \\
Stator tooth pitch $(\mathrm{mm})$ & 15.5 \\
Translator tooth pitch & 10 \\
No. of turns in the copper coil (turns) & 200 \\
Translator tooth width $(\mathrm{mm})$ & 4.5 \\
Translator slot width $(\mathrm{mm})$ & 5.5 \\
Load resistance $(\Omega)$ & 10 \\
Velocity of translator $(\mathrm{m} / \mathrm{s})$ & 1 \\
Air gap $(\mathrm{mm})$ & 1 \\
\hline \hline
\end{tabular}

\section{The Proposed Algorithm}

The random populations can be initialized for the proposed HIGA method. In the beginning, four translator shapes and four stator shapes are considered. The generalized name of the translator shape has been given as "Translator design $n$ " where $n$ denotes the translator design number. Various combinations of stator and translator sets are possible which have been at first permitted for the HIGA to optimize the parameters, such as number of turns in the copper windings, tooth pitch, and gaps between teeth. Among all the combinations, some suitable pairs are verified by the simulation and selected for the subsequent stages.

The flux density, distribution of flux lines in the stator and translator cores, magnetic flux lines inside permanent magnets, and generated voltage and power are analyzed. The path for magnetic flux is designed considering the portions liable to restrict magnetic flux. The designs are used in the next populations in which greater fitness is expected to achieve.

These pairs have been once more passed to the proposed HIGA to achieve higher fitness. This process is carried out until acceptable outcome is obtained. The block diagram of the proposed HIGA method is shown in Fig. 4. The local genetic algorithm inside HIGA optimizes the general parameters without the shapes of the stator and translator. The general parameters are pole-tooth gap, length, pitch, depth and air gap length, which are passed in the local genetic algorithm one by one until satisfactory results are obtained.

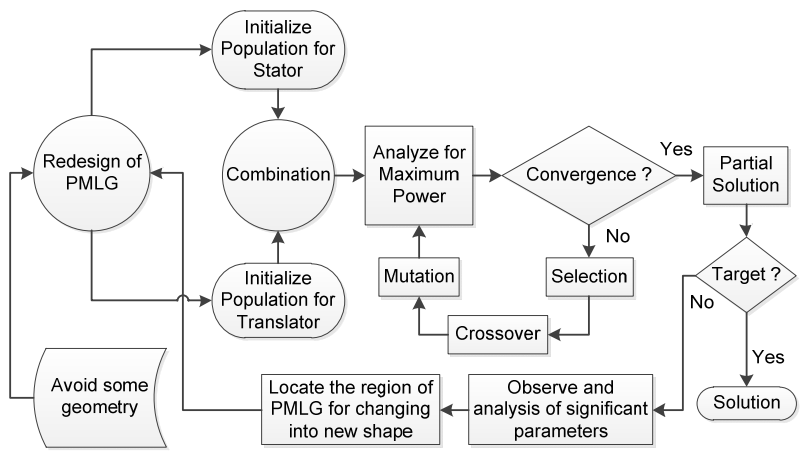

Fig. 4. The proposed HIGA method for shape optimization.

The single segment of the special m-shaped stator core curvature can be represented as follows.

$$
C_{B}(x)=\sum_{k=0}^{m}{ }^{m} C_{k}(1-x)^{m-k} x^{k} Q_{k}
$$

where $x(0 \leq x \leq 1)$ describes the distance of $C_{B}(x)$ from the control point of $Q_{k}$ to the next control point $Q_{k+1}, C_{B}$ represents the locus of the Bezier curve, $Q_{k}$ is the control point, $k$ is the number of control points, and $m$ indicates the number of segments or degree representing the curve. To increase the order of the curve from $m$ to $m+1$, the number of control points needs to vary from $m+1$ to $m+2$, and the new control point $Q_{k}^{m+1}$ can be expressed as follows.

$$
Q_{k}^{m+1}=\frac{k}{k+1} Q_{k-1}^{m}+\left(1-\frac{k}{k+1}\right) Q_{k}^{m}
$$

where $Q_{-1}^{m}=Q_{k+1}^{m}=0, k=0,1, \ldots m+1$ and the shape of the previous segment remains unchanged, To increase the order of the curve from $m$ to $m+n$, the new control point $Q_{k}^{m+n}$ can be expressed as:

$$
Q_{k}^{m+n}=\sum_{j=\max (0, k-n)}^{\min (m, 1)} \frac{{ }^{m} C_{j}{ }^{n} C_{k-j}}{{ }^{m+n} C_{k}} Q_{k}^{m}
$$

where $k=0,1, \ldots, m+n$, and it permits to increase the degree of the curve and the number of control points without changing the shape of the curve. If $Q_{k}+x_{k}=x_{k+1}$, the continuity conditions for the $k^{\text {th }}$ and $k+1^{\text {th }}$ segments of the composite Bezier curve can be stated as:

$$
P_{k+1}\left(Q_{m k}-Q_{m k-1}\right)=P_{k}\left(Q_{m k+1}-Q_{m k}\right)
$$

and 


$$
\begin{aligned}
& Q_{n k-1}+\frac{P_{k+1}}{P_{k}}\left(Q_{m k-1}-Q_{m k-2}\right) \\
& =Q_{n k+1}+\frac{P_{k}}{P_{k+1}}\left(Q_{m k+1}-Q_{m k+2}\right)
\end{aligned}
$$

where $P_{k}$ is the intermediate point between the control points $Q_{k}$ and $Q_{k+1}$. The curvature of the slots between the teeth of the translator can be described by the cubic Bezier curve having four points as follows.

$$
C_{B}(x)=(1-x)^{3} Q_{0}+3 x(1-x)^{2} Q_{1}+3 x^{2}(1-x) Q_{2}+x^{3} Q_{3}
$$

Some of the stator and translator shapes are considered as shown in Fig. 5 for initialization of random population. Fig. 5 also shows the possible combinations of four different shapes of stators and translator designs. The stator-translator pairs with higher output power are considered for the next pass. In the next pass, the segmentations of permanent magnet are analyzed according to the proposed algorithm as shown in Fig. 4.

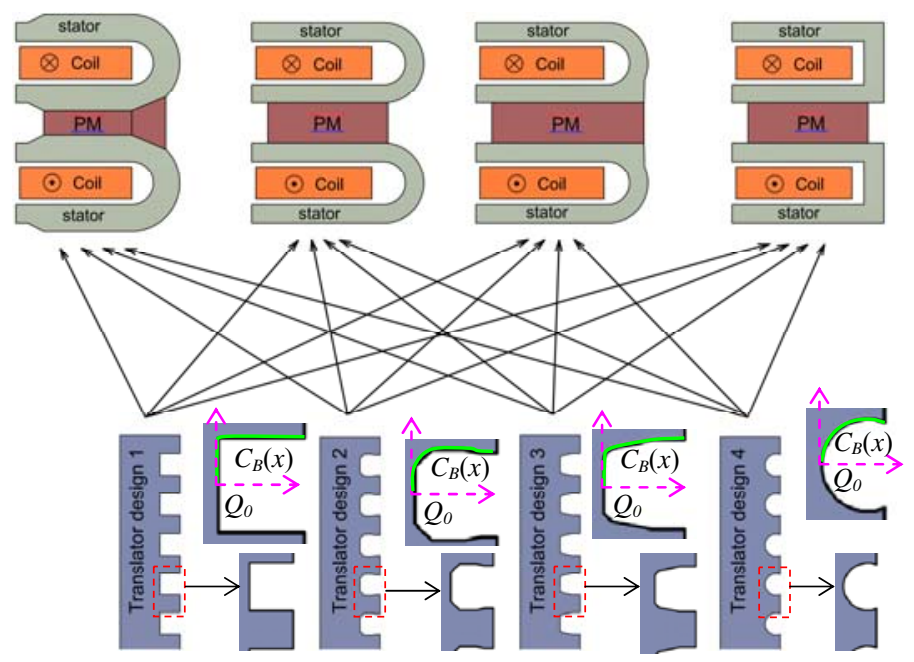

Fig. 5. Combination of the translator and stator pairs.

The generated powers from sixteen combinations of flux switching PMLGs are different due to variations of the permanent magnet shapes. It is necessary to observe the output powers for different shapes of the stator and translator cores with permanent magnets.

The performances of flux switching PMLG for the segmented permanent magnets are analyzed by simulation. Four different shapes of translators and three different shapes of stators with segmented permanent magnets are presented in Fig. 6. Translator designs 5-8 are combined with the stators. The permanent magnet is divided into two or three segments, but the flux switching PMLG containing 2-segmented permanent magnet shows better results than that with 3segmented permanent magnet.

The flux lines and flux density of these combinations are simulated and analyzed. From these analyses it is observed that sharp edges of stator and translator sets are not suitable for proper magnetic flux linkage. The weight of the linear generator can be reduced further by observation of the flux lines to find out the insignificant portions of the stator and/or translator.

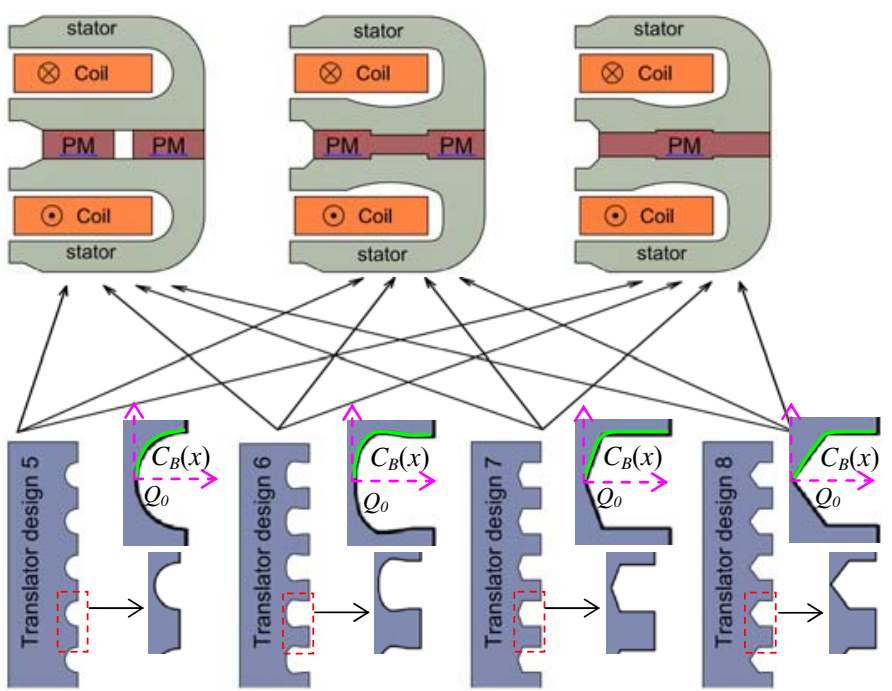

Fig. 6. Some combinations of the translator and stator with segmented permanent magnets.

From the analysis, it is seen that the flux switching PMLG containing segmented permanent magnets cannot generate electricity more than that containing uniform single permanent magnet. For this reason, in the next pass, a single solid permanent magnet will be used with the core having sharp edges. Four modified designs of stators, translators, and their combinations are shown in Fig. 7.

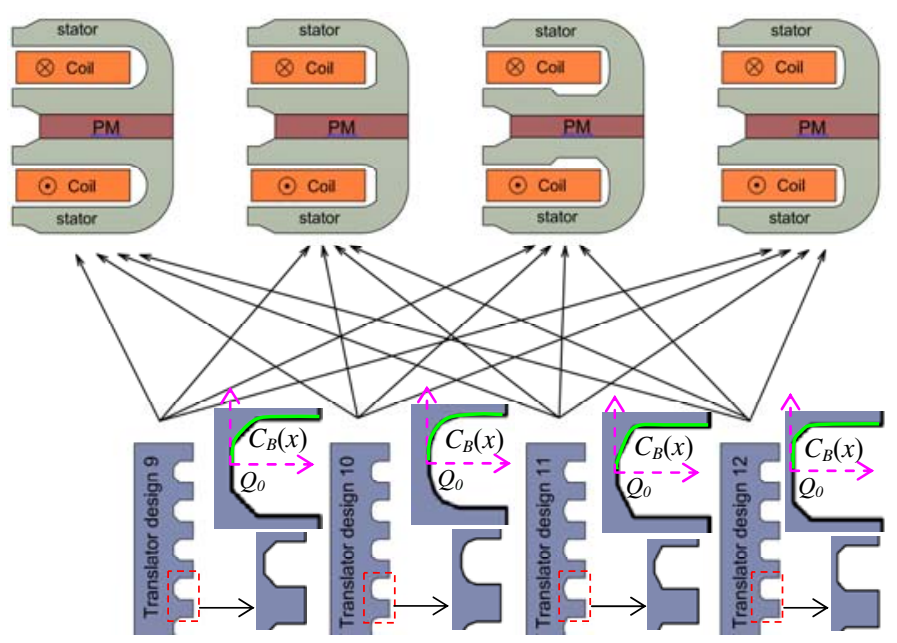

Fig. 7. Combination of the stator and translator with more fitness.

All of the flux switching PMLGs with new combinations can generate more electricity than the previous combinations. From the crossover and mutation after some passes (loops) using the proposed algorithm, it is found that translator designs 13 and 14 have the highest fitness property among various shapes of the translator. Therefore, these two translator shapes as shown in Fig. 8 are chosen for the final design of the proposed flux switching PMLG.

The stator with rounded edge is chosen to combine them 
with the highest fitness of the translator to achieve the best results. Some stator designs with modified shapes are shown in Fig. 8 and their combinations are investigated. Considering various numbers of possible combinations, such as crossover and mutation, the final design is selected according to its best fitness as shown in Fig. 9. Translator designs 13 and 14 are also simulated and same results are obtained. Therefore, these two designs may be considered as equivalent design. The difference between the previously optimized according to [19] and newly optimized using the proposed HIGA method can be realized from Fig. 10. The magnetic flux lines in the same scale of the flux switching PMLG without and with the proposed algorithm are shown for comparison.

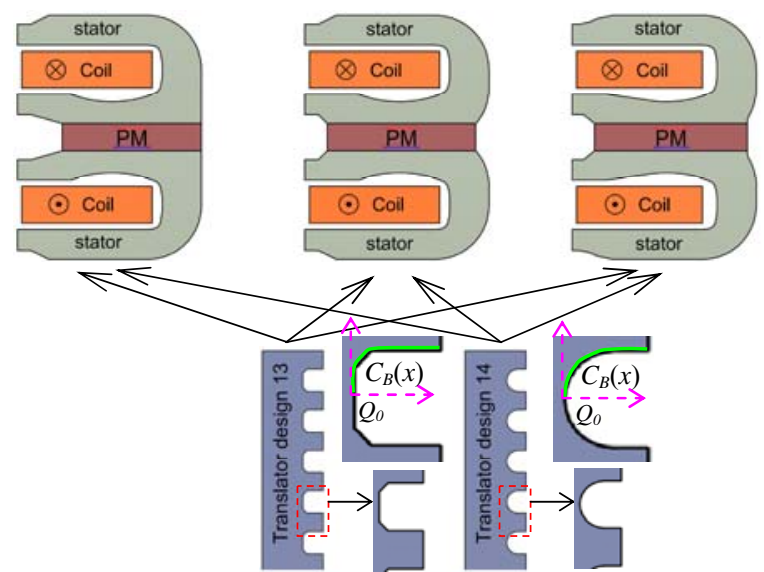

Fig. 8. Combination of the highest fitness of stator with translator designs 13 and 14.

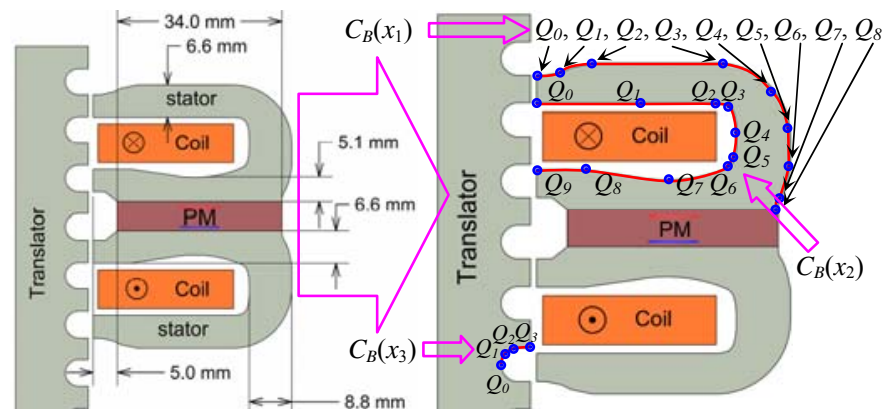

Fig. 9. Cross sectional view of the flux switching PMLG after applying the HIGA method within dimension.

\section{Simulation Results}

In the proposed HIGA method, a goal is needed to set up as the optimization objective, which is to generate $25 \%$ more electrical power from the existing promising flux switching PMLG with the modification of its shape only. Figs. 10 and 11 represent the magnetic flux lines, $A$ and flux density, $B$ of this generator, respectively.

It is found from Figs. 10 and 11 that some regions of iron cores indicated by black ellipse are unnecessary to conduct magnetic flux. Therefore, these regions can be minimized. Similarly, in Fig. 11 some regions of iron cores indicated by red ellipse have conducted less magnetic flux which can be realized from its lower value of $B$. Inside the iron cores in Figs. 10 and 11 indicated by gray ellipses, the uneven distribution of magnetic flux lines should be revised. As a result, all these concerns are considered to change its shape to obtain new improved population. Several new generations yield the suitable shape of flux switching PMLG with high magnetic performance.
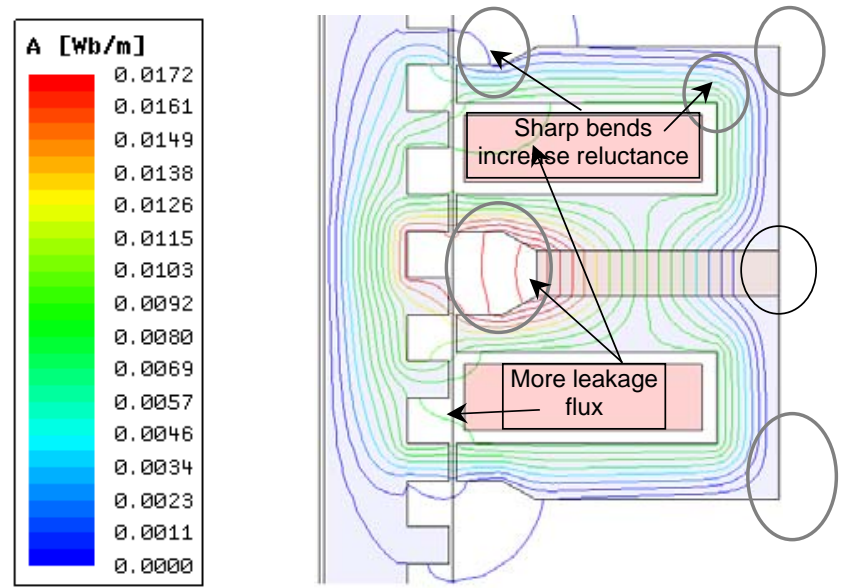

Fig. 10. Magnetic flux lines in the existing flux switching PMLG.
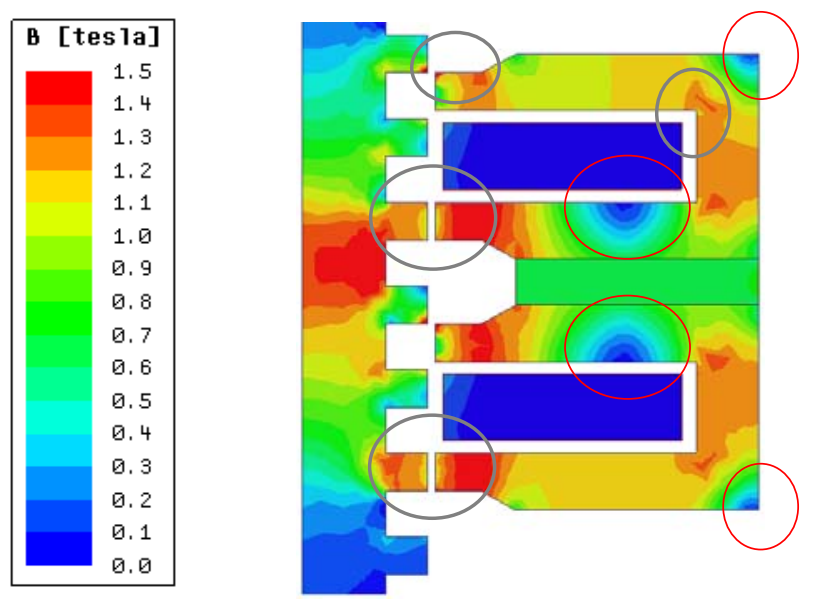

Fig. 11. Magnetic flux density in the existing flux switching PMLG.

The magnitudes of $B$ and $A$ of the designated flux switching PMLG are shown in Figs. 12 and 13, correspondingly in which the ellipses have been indicated to make comparison with Figs. 10 and 11. The selected shape found from the proposed HIGA method can be defined by composite Bezier curves and can also be reproduced. 


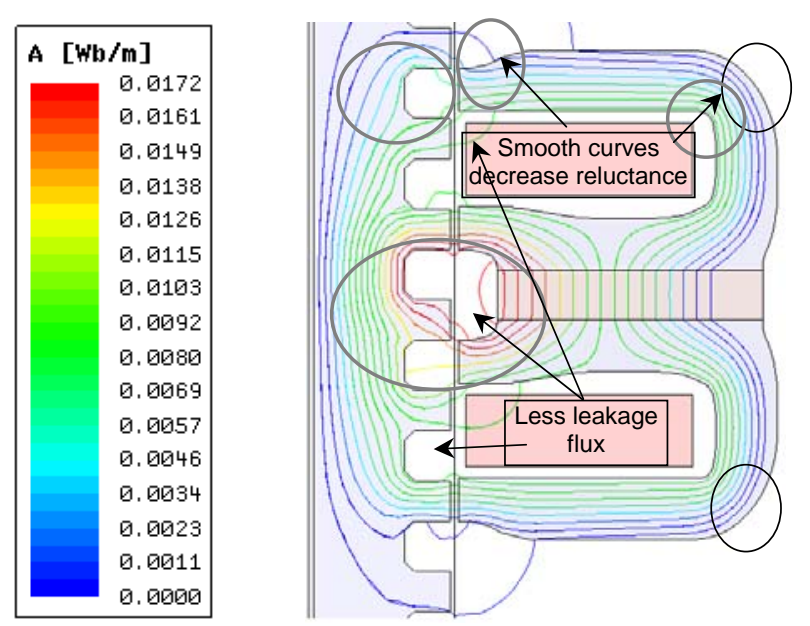

Fig. 12. Magnetic flux lines in the core of optimized flux switching PMLG.
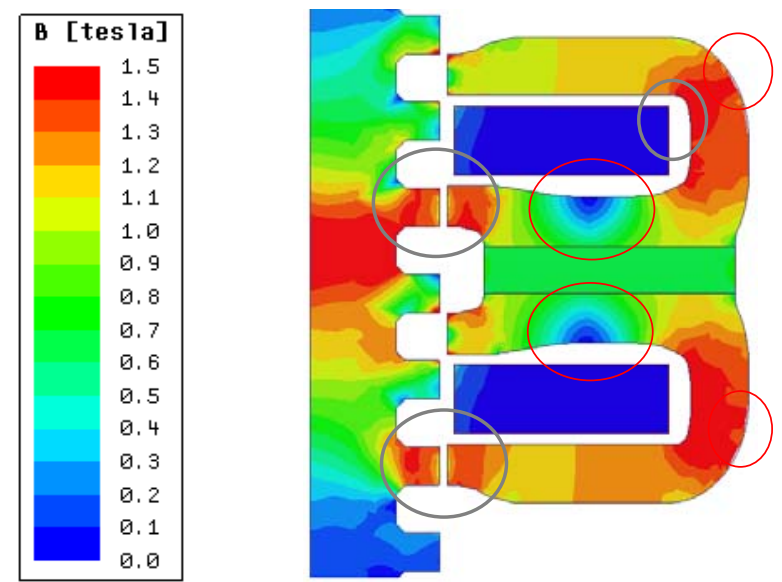

Fig. 13. Magnetic flux density in the core of optimized flux switching PMLG.

From Figs. 12 and 13, it is understood that, in the specific areas enclosed by ellipses, the magnetic flux travels smoothly and a greater value of $B$ would increase electrical power output. The eddy current and hysteresis losses in the flux switching PMLG, before and after implementation of the proposed HIGA method, are shown in Figs. 14. The total core losses in a segment of the flux switching PMLG, before and after implementation of the proposed method, are shown in Figs. 15. The core losses of the proposed design are low compared to the existing one for the same amount of electrical power generation.

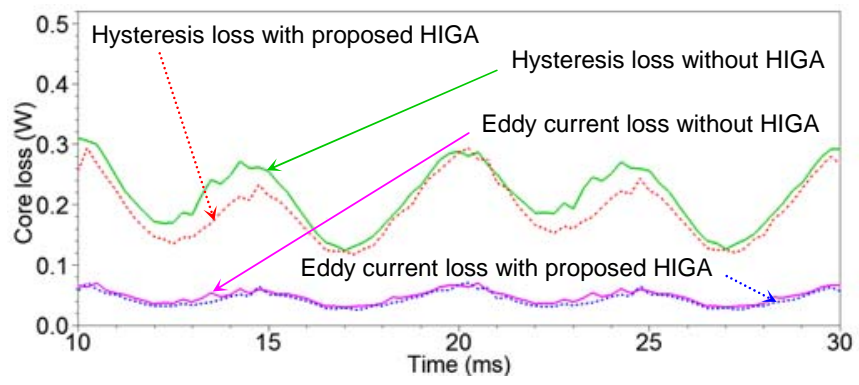

Fig. 14. Eddy current and hysteresis loss for the conventional and the proposed flux switching PMLGs.

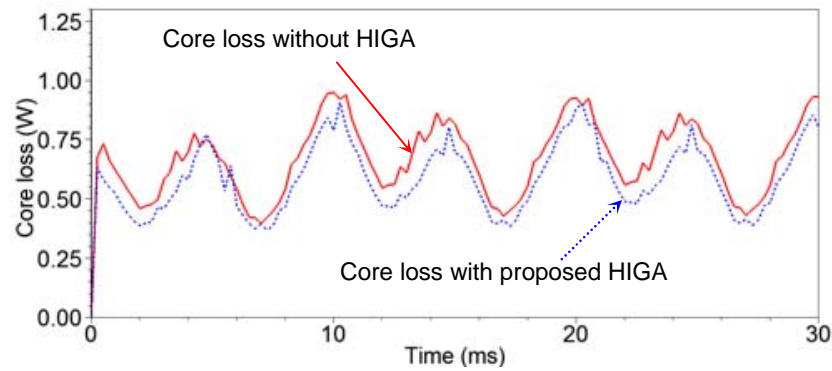

Fig. 15. Core losses for the proposed and existing flux switching PMLGs.

Fig. 16 represents the terminal voltage, load current, and flux linkage of the proposed flux switching PMLG. Comparison of the terminal voltages and flux linkages are shown in Fig. 17. The comparison of the applied forces to the translator of the existing and the proposed flux switching PMLGs are shown in Fig. 18. The comparison of the output electrical powers of the existing and the proposed flux switching PMLGs are shown in Fig. 19 for the same size, shape, and dimensions of permanent magnets. Fig. 19 also shows that improper Bezier curve results in producing lower electrical power rather than increasing electrical power. The electrical power output of the optimized flux switching PMLG is increased by $26.6 \%$.

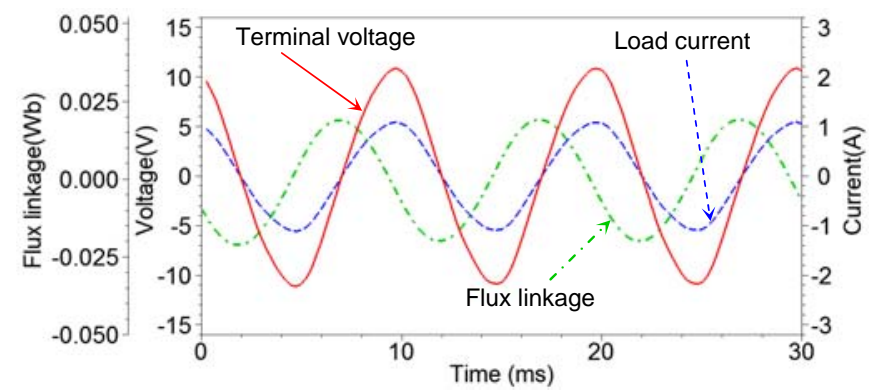

Fig. 16. Terminal voltage, load current, and flux linkage of the proposed flux switching PMLG.

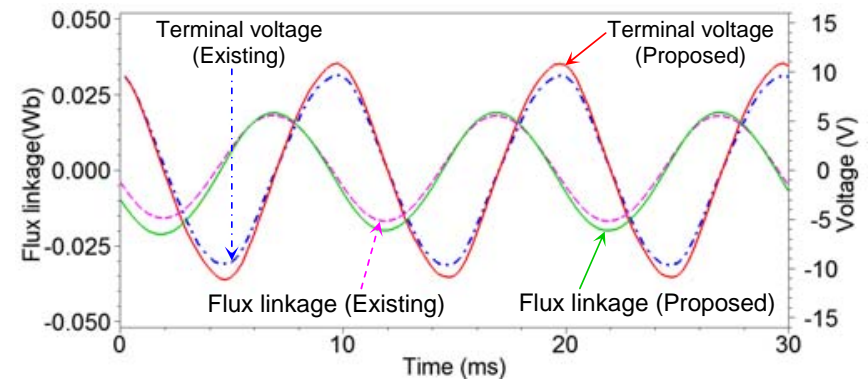

Fig. 17. Comparison of terminal voltages and flux linkages.

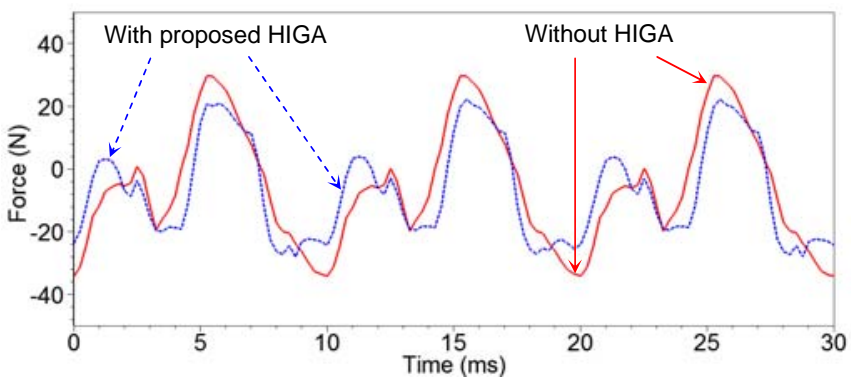

Fig. 18. Comparison of the applied forces. 


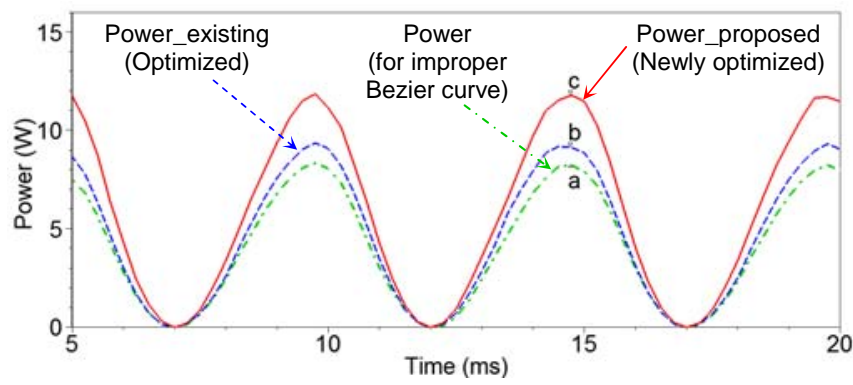

Fig. 19. Comparison of the output electrical powers.

Different parameters and dimensions of the flux switching PMLG without and with applying the HIGA method are tabulated in Table II.

TABLE II

COMPARISON OF DIFFERENT PARAMETERS

\begin{tabular}{lcc}
\hline \hline \multicolumn{1}{c}{ Name of the parameter } & Without HIGA & With HIGA \\
\hline Stator surface area (front view) $\left(\mathrm{mm}^{2}\right)$ & 568.6 & 487.96 \\
Stator volume (cc) & 56.86 & 48.79 \\
Maximum/peak power (W) & 9.13 & 11.78 \\
Peak voltage (V) & 9.65 & 10.81 \\
Load resistance $(\Omega)$ & 10 & 10 \\
\hline \hline
\end{tabular}

\section{CONCLUSION}

By applying the proposed HIGA method it is observed that, the optimized flux switching PMLG can produce nearly $26.6 \%$ more electrical energy compared to the well-known existing one. The proposed method can minimize the size of the iron cores of the special m-shaped stator by $9.06 \%$. In other words, the weight of the proposed flux switching PMLG is lower for the same power rating. Therefore, the multiobjectives can be achieved by using HIGA method. The effectiveness of the proposed m-shaped stator shape can be understood by observing and comparing the gradual distribution of the magnetic flux lines and flux density visually. The finite element method is used to analyze the electrical and magnetic property of this generator. The application of HTS instead of $\mathrm{Cu}$ conductors can further improve the electrical power generation noticeably of this generator. The simulation results exhibit excellent features of the HIGA method applied on the flux switching PMLG as an example, but this method is applicable to other linear generators.

\section{REFERENCES}

[1] M. C. Di Piazza and M. Pucci, "Induction-machines-based wind generators with neural maximum power point tracking and minimum losses techniques," IEEE Trans. Ind. Electron., vol. 63, no. 2, pp. 944955, Feb. 2016

[2] M. R. Islam, Y. G. Guo, J. G. Zhu, H. Y. Lu, and J. X. Jin, "Highfrequency magnetic-link medium-voltage converter for superconducting generator-base high power density wind generation systems," IEEE Trans. Appl. Superconduct., vol. 25, no. 5, article 5202605, Oct. 2014.

[3] M. R. Islam, A. M. M. Rahman, M. M. Islam, Y. G. Guo, and J. G. Zhu, "A modular medium voltage grid connected converter with improved switching techniques for solar photovoltaic systems," IEEE Trans. Ind. Electron., article in press, DOI 10.1109/TIE.2017.2652402.

[4] B. Singh and V. Rajagopal, "Neural-network-based integrated electronic load controller for isolated asynchronous generators in small hydro generation," IEEE Trans. Ind. Electron., vol. 58, no. 9, pp. 4264-4274, Sept. 2011.

[5] M. R. Islam, Y. G. Guo, and J. G. Zhu, "An amorphous alloy core medium frequency magnetic-link for medium voltage photovoltaic inverters," J. Appl. Phy., vol. 115, no. 17, pp. 17E710-1-3, May 2014.
[6] M. R. Islam, Y. G. Guo, and J. G. Zhu, "A high-frequency link multilevel cascaded medium-voltage converter for direct grid integration of renewable energy systems," IEEE Trans. Power Electron., vol. 29, no. 8, pp. 4167-4182, Aug. 2014.

[7] M. R. Islam, G. Lei, Y. G. Guo, and J. G. Zhu, "Optimal design of highfrequency magnetic-links for power converters used in grid connected renewable energy systems," IEEE Trans. Magn., vol. 50, no. 11, article 6971577, Nov. 2014.

[8] M. Alberdi, M. Amundarain, A. J. Garrido, I. Garrido, and F. J. Maseda, "Fault-ride-through capability of oscillating-water-column-based wavepower-generation plants equipped with doubly fed induction generator and airflow control," IEEE Trans. Ind. Electron., vol. 58, no. 5, pp. 1501-1517, May 2011.

[9] E. Tedeschi and M. Molinas, "Tunable control strategy for wave energy converters with limited power takeoff rating," IEEE Trans. Ind. Electron., vol. 59, no. 10, pp. 3838-3846, Oct. 2012.

[10] R. Vermaak and M. J. Kamper, "Design aspects of a novel topology aircored permanent magnet linear generator for direct drive wave energy converters," IEEE Trans. Ind. Electron., vol. 59, no. 5, pp. 2104-2115, May 2012.

[11] I. Stamenkovic, N. Milivojevic, N. Schofield, and M. Krishnamurthy, "Design, analysis, and optimization of ironless stator permanent magnet machines," IEEE Trans. Power Electron., vol. 28, no. 5, pp. 2527-2538, May 2013.

[12] J. Zhang, H. Yu, Q. Chen, M. Hu, L. Huang, and Q. Liu, "Design and experimental analysis of ac linear generator with Halbach PM arrays for direct-drive wave energy conversion," IEEE Trans. Applied Supercon., vol. 24, no. 3, Jun. 2014.

[13] E. Abraham and E. Kerrigan, "Optimal active control and optimization of a wave energy converter," IEEE Trans. Sustain. Energy, vol. 4, no. 2, pp. 324-332, Apr. 2013.

[14] R. Guanche, V. Gómez, C. Vidal, and I. Eguinoa. "Numerical analysis and performance optimization of a submerged wave energy point absorber," Ocean Engineering, vol. 59, pp. 214-230, Feb. 2013.

[15] R. Vermaak and M. J. Kamper, "Design aspects of a novel topology aircored permanent magnet linear generator for direct drive wave energy converters," IEEE Trans. Ind. Electron., vol. 59, no. 5, pp. 2104-2115, May 2012.

[16] A. Pirisi, M. Mussetta, G. Gruosso, and R. Zich, "Optimization of a linear generator for sea-wave energy conversion by means of a hybrid evolutionary algorithm," in Proc. IEEE CEC, 2010, pp. 1-6.

[17] C. Liu, H. Yu, M. Hu, Q. Liu, S. Zhou, and L. Huang, "Research on a permanent magnet tubular linear generator for direct drive wave energy conversion," IET Renew. Power Gener., vol.8, no.3, pp.281-288, Apr. 2014.

[18] L. Huang, H. Yu, M. Hu, C. Liu, and B. Yuan, "Research on a tubular primary permanent-magnet linear generator for wave energy conversions," IEEE Trans. Magn., vol.49, no.5, pp.1917-1920, May 2013.

[19] L. Huang, H. Yu, M. Hu, J. Zhao, Z. Cheng, “A novel flux-switching permanent-magnet linear generator for wave energy extraction application," IEEE Trans. Magn., vol. 47, no. 5, pp.1034-1037, May 2011.

[20] J. A. Tapia, J. Pyrhonen, J. Puranen, P. Lindh, and S. Nyman, "Optimal design of large permanent magnet synchronous generators," IEEE Trans. Magn., vol. 49, no. 1, pp. 642-650, Jan. 2013.

[21] J. A. Tapia, A. Parviainen, J. Pyrhönen, P. Lindh, and R. R. Wallace, "Optimal design procedure for an external rotor permanent-magnet machine," in Proc. XXth Int. Conf. Electric. Mach., Marseille, 2012, pp. 2695-2701.

[22] G. Bramerdorfer, A. C. Zăvoianu, S. Silber, E. Lughofer, and W. Amrhein, "Possibilities for speeding up the FE-based optimization of electrical machines-A case study," IEEE Trans. Ind. Appl., vol. 52, no. 6, pp. 4668-4677, Nov.-Dec. 2016.

[23] G. Bramerdorfer, W. Amrhein, S. M. Winkler, and M. Affenzeller, "Identification of a nonlinear PMSM model using symbolic regression and its application to current optimization scenarios," in Proc. $40^{\text {th }}$ Annu. Conf. IEEE Ind. Electron. Society, Dallas, USA, 2014, pp. 628-633.

[24] G. Bramerdorfer, S. Silber, G. Weidenholzer, and W. Amrhein, "Comprehensive cost optimization study of high-efficiency brushless synchronous machines," in Proc. Int. Electric Mach. Drives Conf., Chicago, IL, 2013, pp. 1126-1131.

[25] R. Nasiri-Zarandi, M. Mirsalim, and A. Cavagnino, "Analysis, optimization, and prototyping of a brushless de limited-angle torque- 
motor with segmented rotor pole tip structure," IEEE Trans. Ind. Electron, vol. 62, no. 8, pp. 4985-4993, Aug. 2015.

[26] O. Farrok, M. R. Islam, M. R. I. Sheikh, and W. Xu, "A new optimization methodology of the linear generator for wave energy conversion systems," in Proc. IEEE Int. Conf. Ind. Techn., Taipei, Taiwan, 2016, pp. 1412-1417.

[27] O. Farrok, M. R. Islam, M. R. I. Sheikh, Y. Guo, J. Zhu, and W. Xu, “A novel superconducting magnet excited linear generator for wave energy conversion system," IEEE Trans. Applied Supercon., vol. 26, no. 7, art. 6207105, Oct. 2016. 